\title{
A proposal for benchmark tests for underactuated or compliant hands
}

\author{
G. A. Kragten, C. Meijneke, and J. L. Herder \\ Faculty of Mechanical, Maritime and Materials Engineering, Dept. of BioMechanical Engineering, \\ Delft University of Technology, Mekelweg 2, 2628 CD Delft, The Netherlands
}

Received: 25 February 2010 - Revised: 15 June 2010 - Accepted: 19 August 2010 - Published: 22 December 2010

\begin{abstract}
There is a lack of agreement in the literature as to what exactly quantifies the performance of underactuated hands. This paper proposes two benchmark tests to measure the ability of underactuated hands to grasp different objects and the ability to hold the objects when force disturbances apply. The first test determines the smallest and largest cylindrical object which can be successfully grasped in an enveloping grasp or in a pinch grasp. The second test provides the maximal allowable force which can be applied to a grasped object without loosing it. A setup was constructed consisting of standard components. Exemplary tests were applied to the Delft Hand 2. The proposed benchmark tests are representative to quantify the performance of pick and place operations with underactuated hands. The results of the tests can be applied to evaluate, compare, and improve the performance of robotic hands.
\end{abstract}

This paper was presented at the IFToMM/ASME International Workshop on Underactuated Grasping (UG2010), 19 August 2010, Montréal, Canada.

\section{Introduction}

The need for adaptive grasping, the reduction of actuators, weight, costs, and the reduction of control complexity are reasons to choose for underactuated robotic hands instead of fully actuated ones (e.g. Laliberté and Gosselin, 1998; Wu et al., 2009). Prototypes presented in the literature show that such hands are indeed able to grasp a large variety of objects, while the hand is driven even with a single motor (e.g. Gosselin et al., 2008). However, comparison of the performance of these prototypes based on the literature is almost impossible. Actually, there is hardly any agreement about the definition of performance. This means that the proposal of future research directions or design improvements substantiated by the results presented in literature seems impossible.

In previous research (Kragten and Herder, 2010), we made an overview of performance metrics which were applied to the design or evaluation of underactuated hands. Based on these metrics, two new, functional metrics were compiled to quantify the performance to pick and place different ob-

Correspondence to: G. A. Kragten (g.a.kragten@tudelft.nl) jects. They firstly quantify the range of object sizes that can be successfully grasped by an underactuated hand (ability to grasp), and secondly quantify the allowable force disturbances on the grasped objects (ability to hold). Cylindrically shaped objects were used in the definitions, because such objects are easy for parameterization, for contact modeling, and for reproduction in experiments. In addition, the shape of many objects in daily life can be approximated by a cylinder (van Lunteren and van Lunteren-Gerritsen, 1989). A static grasp model was presented in (Kragten and Herder, 2010) to calculate these performance metrics. However, a test to measure these performance metrics for prototypes of underactuated hands does not exist.

The objective of this paper is to propose benchmark tests to assess the performance of underactuated or compliant hands. It was aimed to define tests that are easily reproducible, that only need standard components, and quantify the performance regarding the ability to grasp and to hold objects. The results of such tests can be used to compare the performance of underactuated or compliant hands, and to measure the effect of design modifications. The tests proposed in this paper are limited to planar grasping but allow extension to spatial grasps. 


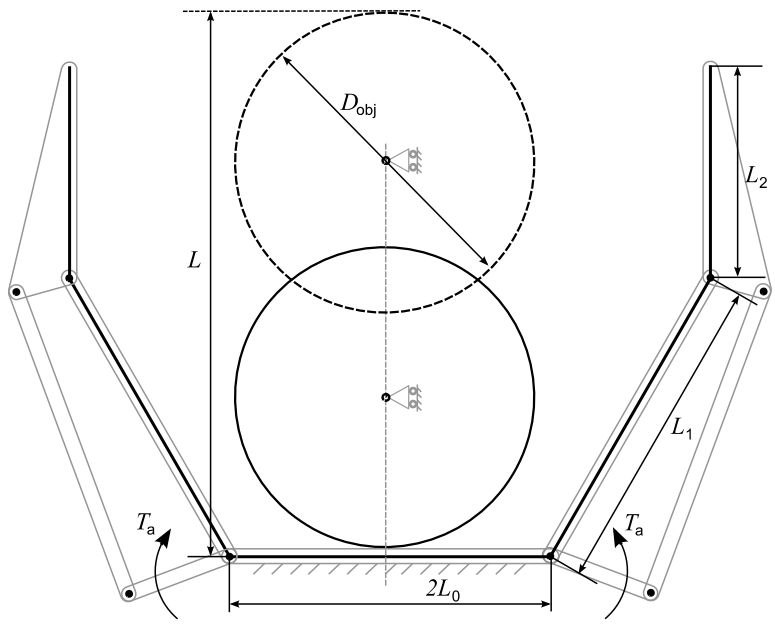

Figure 1. Initial positions of a freely moving cylindrical object with respect to the palm of a hand to determine the ability to successfully grasp this object. The palm position is represented by the solid object, and the pinch position by the dashed object. $D_{\text {obj }}$ is the diameter of the object, $2 L_{0}$ is the width of the palm, $L_{1}$ is the length of the proximal phalanx, $L_{2}$ is the length of the distal phalanx, $L$ is the length of the finger. A torque $T_{\mathrm{a}}$ applies to the base of the fingers.

The structure of the paper is as follows. First a benchmark test to measure the range of objects which can be successfully grasped is proposed. In this test, grasping by enveloping or pinching the objects is distinguished. Then a benchmark test to measure the maximum allowed force disturbances applied to a grasped object is proposed. A setup to perform both tests is described, on which experiments with the Delft Hand 2 (DH-2) (Meijneke et al., 2010) are executed. The results of these tests are presented and discussed.

\section{Methods}

In the following sections, the benchmark tests and the experimental setup are defined.

\subsection{Benchmark test 1: ability to grasp objects}

It was proposed to quantify the range of object sizes that can be successfully grasped by the smallest and largest cylinder for which grasp equilibrium exists (Kragten and Herder, 2010). The performance metric was defined as follows:

$Q_{\text {grasp }}=\frac{\frac{\pi}{2} \Delta D_{\text {obj }}}{2 L+2 L_{0}}$

where $\Delta D_{\text {obj }}$ is the difference between the diameter of the largest and smallest graspable object, $L=L_{1}+L_{2}$ is the length of one finger and $2 L_{0}$ the palm width of the hand, see also Fig. 1. This metric can assume values between 0 and 1 for frictionless grasping, where 1 is regarded as the best performance. To measure this performance, freely moving, cylin- drical objects of various sizes are grasped to assess the smallest and largest size for which grasp equilibrium exists. We propose to distinguish between an enveloping grasp where the object is enclosed by the fingers and near the palm, and a pinch grasp where the object is grasped by the distal phalanges only. This distinction was made, because objects which have to be grasped from the top normally have to be grasped by the distal part of the fingers. Also smaller objects are more likely to be grasped by the distal phalanges while large objects are more likely to be enveloped by the fingers.

The proposed benchmark test is as follows. Place a rigid cylinder with a radius $D_{\text {obj }}$ on a surface or platform with low friction. Position the palm of the hand against the object for enveloping grasping - or on a distance $L$ from the object - for pinch grasping, while the fingers are in a fully open configuration, see Fig. 1. Apply a constant torque to actuate the fingers and to grasp the object. Determine if the object is successfully grasped (e.g. equilibrium of the fingers and the object). Repeat the test with different sizes of cylinders to determine the smallest and largest object for which a enveloping grasp or pinch grasp exist.

For this test, cylinders in the sizes of $40,50,55,60,63$, $75,90,110,115,120 \mathrm{~mm}$ diameter were available. Currently, only experiments with symmetric hands were conducted. Therefore, freely moving of the objects along the line of symmetry was sufficient, which was realized by a linear guide (THK, RSR 9KM).

\subsection{Benchmark test 2: ability to hold objects}

It was proposed to determine the minimal force needed to pull a grasped object all the way out of the hand in order to quantify the maximal allowable external forces on the object (Kragten and Herder, 2010). The performance metric was defined as follows:

$Q_{\text {hold }}=\frac{F}{T_{\mathrm{a}} / L}$

where $F$ is the minimal force needed to pull a object out of the hand, $T_{\mathrm{a}}$ is the constant actuation torque applied at the base of the fingers as defined in Birglen et al. (2008, p. 35), and $L$ is the total length of the finger. A high value of $Q_{\text {hold }}$ is regarded as a good performance. The force needed to pull an object out of the hand depends on the pull direction. Therefore, different disturbance directions have to be tested. The worst-case direction determines the maximum allowed external force on the object.

The proposed benchmark test is as follows. Place a cylinder with a diameter $D_{\text {obj }}$ against the palm of the hand. Slowly move the object along a straight line in the disturbance direction $u$, and allow the object to move in the perpendicular direction $v$, see Fig. 2. Measure the force needed to pull the object and determine the maximum force along this trajectory. Repeat this measurement for increasing $\Phi$. Determine the minimum of the maxima of all the force-displacement characteristics. 


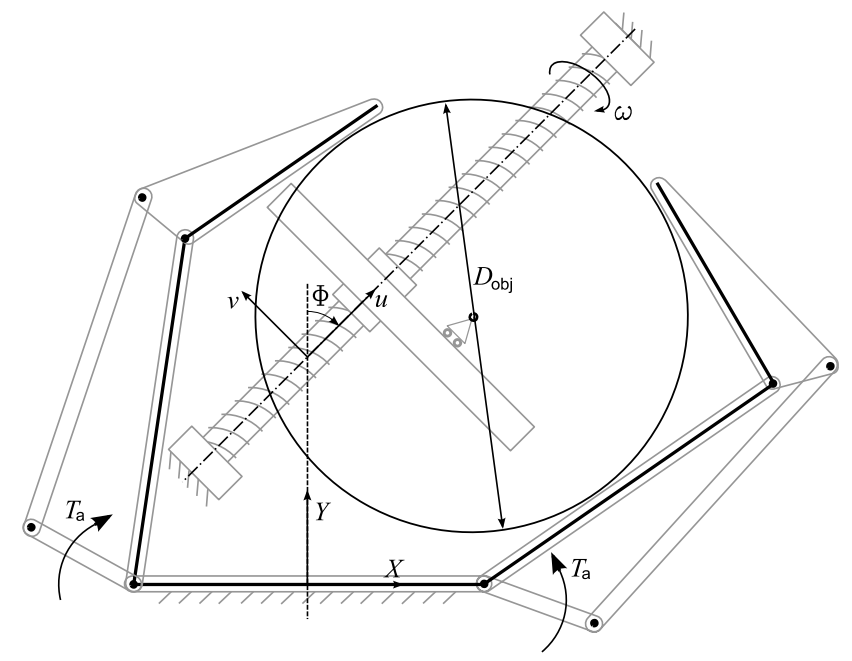

Figure 2. Schematic drawing of the setup where a cylindrical object with a diameter $D_{\text {obj }}$ is pulled out of the hand at a constant slow speed $\omega$ in the direction $u$, while the fingers are actuated at a constant torque $T_{\mathrm{a}}$. The object is free to move in the direction $v$, which is perpendicular to $u$. The resultant of the contact forces on the object in the pull direction $u$ is measured.

A setup was built for the benchmark test to determine the minimal force needed to pull the object out of the hand. A picture of this setup is shown in Fig. 3. The setup consists of a turning table (1), where $\Phi$ can be adjusted between -90 and 90 degrees in steps of 15 degrees. On top of this turning table, a linear ball screw spindle (2) is mounted (Hiwin, $\mathrm{KK} 40$, pitch $1 \mathrm{~mm}$, stroke $200 \mathrm{~mm}$ ), which is driven at a constant speed of $5 \mathrm{~mm} / \mathrm{s}$ by a brushed motor (3) (Maxon, AMax26). The speed was controlled by a universal motion interface (National Instruments, UMI-7772) and LabView 8.2 on a standard PC. A linear guide (4) (THK, RSR 9KM) was mounted perpendicular to the spindle. The force sensor (5) (FUTEK, LSB200, capacity $110 \mathrm{~N}$ ) was placed on top of this guide to eliminate measuring the friction in the linear drive. This sensor measured only the resultant of the contact forces that were applied to the object in the direction of the linear drive. All bending moments and forces in other directions on the object were supported by a construction of leaf springs. On top of this force sensor, objects of different size can be mounted. The position of the object in the pull direction was measured by the encoder of the motor. The perpendicular position was optically measured by a laser sensor (6) (optoNCDT, ILD1300-100) that moved along with the linear guide. The force and position data were collected at a sample rate of $20 \mathrm{~Hz}$.

\subsection{Experiments with the Delft Hand 2}

By way of example, the benchmark tests were used to quantify the performance of the Delft Hand 2 (DH-2). This hand consists of three underactuated fingers each having 2 pha-

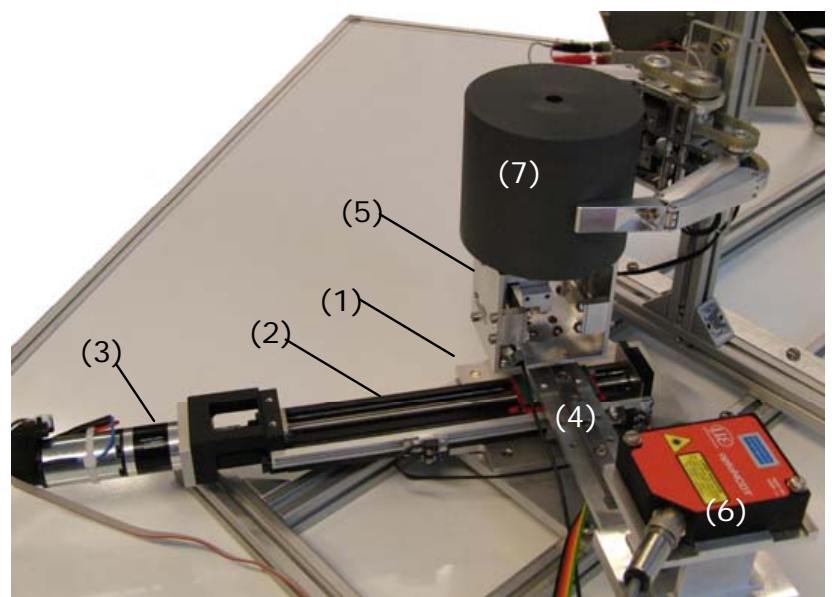

Figure 3. Photo of the setup for benchmark test 2 where (1) is a turning table, (2) is a ball screw spindle, (3) is the motor to drive the spindle; (4) is a linear guide perpendicular to the spindle; (5) is the force sensor supported by a construction of leaf springs; (6) is an optical displacement sensor; and (7) is a cylindrical object.

langes. The driving mechanism of the finger is inspired by the four bar linkage of the SARAH (Birglen et al., 2008, Ch. 7). More details about the design and construction of this hand can be found in a companion paper (Meijneke et al., 2010).

First the range of objects that could be grasped by this hand (benchmark test 1) was measured. Performance calculations comparable to those presented in (Kragten and Herder, 2010) predicted that objects with a diameter between 58 and $130 \mathrm{~mm}$ could be grasped in an enveloping grasp type. It was predicted that the fingers would even pull the object towards the palm and have equilibrium in an enveloping grasp when the objects were initially located at a finger length distance from the palm.

The ability to hold (benchmark test 2) was measured for an object with $\varnothing 115 \mathrm{~mm}$. The object was pulled a distance of $30 \mathrm{~mm}$ in the directions $\Phi=0^{\circ}, 45^{\circ}, 90^{\circ}$, while the force and the object displacement were measured. All experiments were repeated three times, and the maximum measured force was determined for each test. The waiting time between the experiments was at least $30 \mathrm{~s}$ to minimize the effect of temperature differences of the motor on the performance.

\section{Experimental results}

The smallest graspable object in the Delft Hand 2 (DH2) was $D_{\text {obj,min }}=55 \mathrm{~mm}$. The largest available object of $D_{\text {obj,max }}=120 \mathrm{~mm}$ could also be grasped by the hand. When the objects were located at the pinch grasp position (see Fig. 1), the fingers still pulled the objects towards the palm, and an enveloping grasp resulted. The $Q_{\text {grasp }}$ of the DH-2 is at least 0.39 according to Eq. (1) and the results in Table 1. 


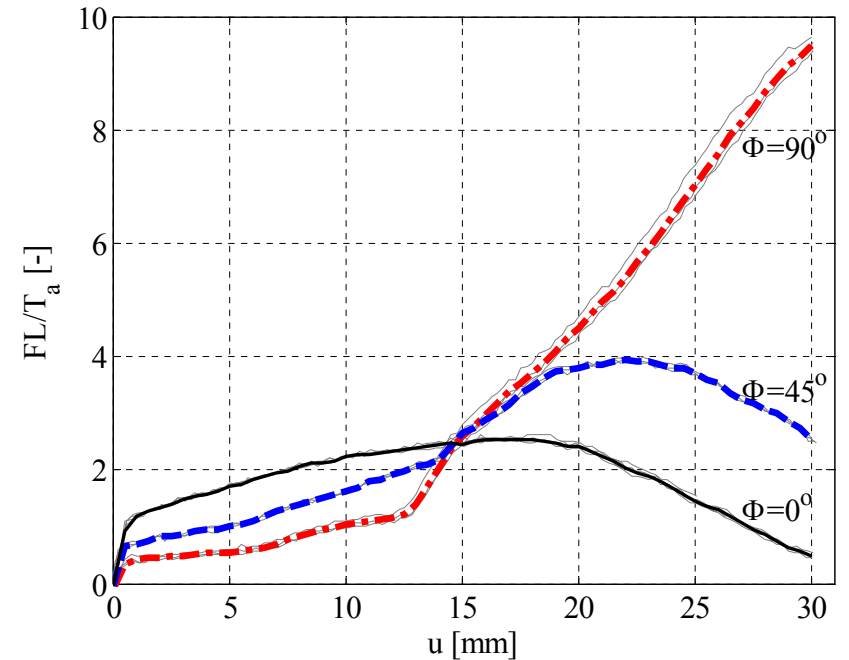

Figure 4a. Mean pull force $F$ as a function of the displacement $u$ of an object $\left(D_{\text {obj }}=115 \mathrm{~mm}\right)$ in three different directions $\Phi$ (see also Fig. 2). $F$ is normalized to the actuation torque $T_{\mathrm{a}}$ and the finger length $L$. The gray, thin lines represent the raw data.

Table 1. Results of benchmark test 1 , showing that in both object positions the smallest and largest available objects could be successfully grasped.

\begin{tabular}{lcc}
\hline & Palm Position & Pinch Position \\
\hline$D_{\text {obj }} \min$ & 55 & 55 \\
$D_{\text {obj }} \max$ & 120 & 120 \\
\hline
\end{tabular}

A better performance could have been measured if larger objects were available. It was observed that objects smaller than $D_{\text {obj,min }}$ were also pulled towards the palm of the hand, but due to the mechanical limits in the joints, the fingers could not maintain contact with the object.

The force-displacement graph of the object of $D_{\mathrm{obj}}=$ $115 \mathrm{~mm}$ pulled in the directions of $\Phi=0^{\circ}, 45^{\circ}, 90^{\circ}$ are shown in Fig. 4a, where the force is normalized by the actuation torque of the finger $\left(T_{\mathrm{a}} \approx 557 \mathrm{Nmm}\right)$ and the length of the finger $(L=100 \mathrm{~mm})$. In addition, the trajectory of the object during the disturbance is shown in Fig. $4 \mathrm{~b}$. The mean of the maximum force of the three repeated experiments was calculated for each disturbance direction and is shown in Table 2. So, the maximum allowed force disturbance on this object is $14.3 \mathrm{~N}$ at the current actuation torque and finger length. This means that, according to Eq. (2), the $Q_{\text {hold }}$ of this hand and object is 2.56 .

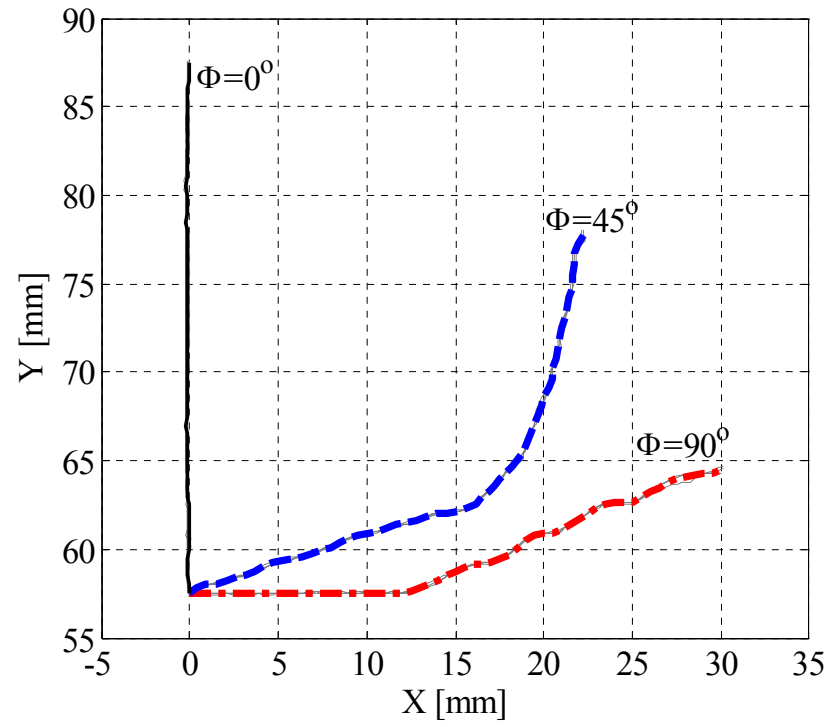

Figure 4b. Mean displacement trajectories in the X-Y-frame of an object $\left(D_{\text {obj }}=115 \mathrm{~mm}\right)$ while it was pulled in three different directions $\Phi$ (see also Fig. 2). $F$ is normalized to the actuation torque $T_{\mathrm{a}}$ and the finger length $L$. The gray, thin lines represent the raw data.

Table 2. Results of benchmark test 2, showing the minimal force needed to extract an object of $D_{\text {obj }}=115 \mathrm{~mm}$ all the way out of the hand in three different directions (standard deviation between brackets). The second line shows the force normalized by the actuation torque $\left(T_{\mathrm{a}}=557 \mathrm{Nmm}\right)$ and finger length $(L=100 \mathrm{~mm})$.

\begin{tabular}{lccc}
\hline & $\Phi=0^{\circ}$ & $\Phi=45^{\circ}$ & $\Phi=90^{\circ}$ \\
\hline$F$ & $14.3 \mathrm{~N}(\sigma=0.08)$ & $22.1 \mathrm{~N}(\sigma=0.15)$ & $53.4 \mathrm{~N}(\sigma=0.27)$ \\
$Q_{\text {hold }}$ & 2.56 & 3.94 & 9.46 \\
\hline
\end{tabular}

\section{Discussion}

Two benchmark tests were proposed in this paper. The first one determines the range of object sizes which can be grasped by an underactuated or compliant hand. The second test measures the minimal force needed to pull the grasped objects out of the hand. The first benchmark test quantifies the adaptability of the hand to grasp different items. The larger this range, the better the hand is capable of grasping various objects, which is useful in unstructured environments. The second benchmark test quantifies the maximal allowable external force that can be applied to the grasped objects in different directions. This force can be interpreted as the maximum allowed weight of the object (payload), or as the maximum acceleration of the robot arm - multiplied by the mass of the object - as a function of the orientation of the hand. Since the measured force was normalized by the actuation torque, the result of the test also shows the effectiveness 
at which the actuation torque is distributed to the phalanges. Finally, the test showed the worst-case disturbance direction. This result might be input for the design to improve the performance of the hand at this scenario, or it might be input for the application of the robot hand to prevent this scenario.

The benchmark tests were applied to the DH-2. The tests could be done with standard components and were simple to execute. The force-displacement characteristics showed a small variation between the experiments. For this hand, pulling the object straight out of the hand is the worst-case scenario. $14.3 \mathrm{~N}$ was required to extract the object, while the fingers were actuated at $T_{\mathrm{a}}=557 \mathrm{Nmm}$. This means, for example, that the weight of an object with $\varnothing 115 \mathrm{~mm}$ must be less than $1.5 \mathrm{~kg}$ when the orientation of the hand is with the fingers vertically downward.

During the design phase of the DH-2, the forcedisplacement characteristics were calculated based on a static, frictionless model (Meijneke et al., 2010). However, the calculated forces were much smaller than the measured results. We observed that friction in the joints strongly limited the extension of the distal phalanges with respect to the proximal phalanges once the fingers have enveloped the object. When the object was pulled out of the hand, the fingers only rotated about the proximal joint. The calculations, however, showed that both joints would rotate if there were no friction in these joints. In addition, contact friction was also neglected in the grasp model. Hence, we assume that the large deviation between the calculated and measured results for the second benchmark test with the DH-2 is mainly caused by neglecting friction in the model. Clearly, friction should not be neglected by default for adequate simulation results.

Rigid, cylindrical objects were used to simplify the reproduction of the tests. The assumption of cylindrical objects is also common in the literature. However, to verify if the results obtained with cylindrical objects are representative, preliminary benchmark tests were executed with square, nonrigid objects of the sizes $52 \times 52,58 \times 58$, and $75 \times 75 \mathrm{~mm}$. For non-cylindrical objects, the orientation has to be specified. The initial chosen orientations of the object were (i) with one side parallel to the palm and (ii) with one corner pointing to the palm. The DH-2 was able to grasp all these objects in both orientations from the palm position and the pinch position. When grasping the smallest square in the orientation parallel to the palm, the fingers were almost at their mechanical limits. This means that the order of magnitude of the size of graspable squares equals the size of cylindrical objects. The force needed to pull the squares out of the hand strongly depended on their initial orientation. This means that the maximum allowed external force on cylindrical objects cannot directly be applied to objects with comparable sizes but other shapes. For future work, the limitations of the use of cylindrical objects to calculate or measure the performance have to be investigated in more detail.
The proposed benchmark tests are limited to planar grasp cases, which is common in the grasping literature. The necessity to extend to spatial tests must be investigated in future work. Furthermore, the proposed benchmark test do not capture other issues like, for instance, the prevention of object damaging, the safety of the hand or the sensitivity of the successful grasp range to initial position deviations of the object (Dollar et al., 2010). Future research is needed to translate the important functional requirements of robotic hands to performance metrics that can be calculated by relatively simple grasp models and measured by tests that can be easily reproduced.

\section{Conclusions}

Two benchmark tests were defined to quantify the performance of underactuated or compliant hands to pick and place different objects. The adaptability of robotic hands to grasp various objects is quantified and tested by measuring the smallest and largest cylindrical object for which a grasp equilibrium exists (benchmark test 1). The maximum allowed weight or external disturbing force on a grasped object is quantified and tested by the minimal force needed to pull the object all the way out of the hand (benchmark test 2).

The benchmark tests are successfully applied to the Delft Hand 2 (DH-2). The tests showed that this hand can grasp objects between at least $\varnothing 55$ and $\varnothing 120 \mathrm{~mm}$ without any feedback control or active adjustment of the hand. The maximal allowed disturbing force on an object of $\varnothing 115 \mathrm{~mm}$ is $14 \mathrm{~N}$ when applied perpendicular to the palm up to $52 \mathrm{~N}$ when applied parallel to the palm, while the fingers were actuated by a constant torque of $0.56 \mathrm{Nm}$. The proposed benchmark tests can be applied to evaluate, compare, and improve the performance of robotic hands.

Acknowledgements. This work was carried out as part of the FALCON project under responsibility of the Embedded System Institute with Vanderlande Industries as the industrial partner. This project is partially supported by the Dutch Ministry of Economic Affairs within the framework of the Embedded System Institute (BSIK03021) program.

We gratefully acknowledge the help of Rik van den Hoeven and Jos van Driel to construct the setup for the experiments.

Edited by: J. L. Herder

Reviewed by: two anonymous referees 


\section{References}

Birglen, L., Laliberté, T., and Gosselin, C. M.: Underactuated Robotic Hands, Springer Tracts in Advanced Robotics, Vol. 40, Springer-Verlag, Berlin, Heidelberg, 2008.

Dollar, A. M., Jentoft, L. P., Gao, J. H., and Howe, R. D.: Contact sensing and grasping performance of compliant hands, Auton. Rob., 28(1), 65-75, 2010.

Gosselin, C. M., Pelletier, F., and Laliberté, T.: An anthropomorphic underactuated robotic hand with 15 dofs and a single actuator, IEEE International Conference on Robotics and Automation ICRA 2008, 749-754, 2008.

Kragten, G. A. and Herder, J. L.: The Ability of Underactuated Hands to Grasp and Hold Objects, Mech. Mach. Theory, 45(3), 408-425, 2010.
Laliberté, T. and Gosselin, C. M.: Simulation and Design of Underactuated Mechanical Hands, Mech. Mach. Theory, 33(1-2), 39-57, 1998.

van Lunteren, A. and van Lunteren-Gerritsen, G. H. M.: On the use of prostheses by children with a unilateral congenital forearm defect, Journal of Rehabilitation Sciences, 2(1), 10-12, 1989.

Meijneke, C., Kragten, G. A., and Wisse, M.: Design and Performance Assessment of an Underactuated Hand for Industrial Applications, Mech. Sci., in press, 2010.

Wu, L., Carbone, G., and Ceccarelli, M.: Designing an underactuated mechanism for a 1 DOF finger operation, Mech. Mach. Theory, 44(2), 336-348, 2009. 